\title{
Detección del gen mecA en cepas de Staphylococcus coagulasa positiva aisladas desde gatos
}

\author{
Nicolás Galarce, Loreto Muñoz, María Antonieta Jara, Paulo Lubí, Alejandra Sepúlveda y Sonia Anticevic
}

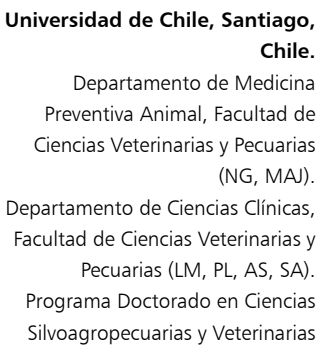

Departamento de Medicina Preventiva Animal, Facultad de Ciencias Veterinarias y Pecuarias (NG, MAJ)

Departamento de Ciencias Clínicas, Facultad de Ciencias Veterinarias y

Pecuarias (LM, PL, AS, SA). Programa Doctorado en Ciencias Silvoagropecuarias y Veterinarias

(NG).

Los autores declaran no poseer conflictos de interés.

Financiamiento: Proyecto FIV No 12101401.9102 .008

Recibido: 29 de marzo de 2016 Aceptado: 18 de julio de 2016

Correspondencia a: Sonia Anticevic C. Fax: 29785606

Casilla 2 Correo 5 La Granja. santicev@uchile.c

\section{Introducción}

L a resistencia antimicrobiana en bacterias es un tema de gran preocupación mundial, comprometiendo a diversos profesionales relacionados a la salud pública, como son los Médicos Veterinarios' ${ }^{1}$. Una de las principales actividades de estos profesionales es la práctica clínica de atención a mascotas, donde diariamente se diagnostican enfermedades infecciosas y se prescriben antimicrobianos. En este contexto, se han publicado diversos reportes de cepas de Staphylococcus resistentes a antimicrobianos aislados desde mascotas ${ }^{2,3}$. Estos agentes son patógenos oportunistas que pueden colonizar la piel y mucosas de personas y animales, y a su vez pueden causar graves enfermedades cutáneas, tisulares o cavitarias ${ }^{4}$.

En Medicina Veterinaria, dos especies de Staphylococcus se consideran como las más relevantes: $S$. aureus y S. intermedius ${ }^{6}$. Sin embargo, en el año 2005 este género fue reclasificado taxonómicamente, considerándose actualmente como $S$. pseudintermedius a la gran mayoría de los aislados de perros y gatos y algunos de equinos ${ }^{6}$. Las pruebas fenotípicas no son de gran ayuda para diferenciar S. intermedius de S. pseudintermedius ${ }^{7}$, por lo que se prefiere considerar a los aislados de $S$. intermedius como pertenecientes al "grupo de S. intermedius" (SIG, por sus siglas en inglés). La virulencia de estas especies se asocia a la producción de coagulasa, por lo que los aislados capaces de producirla son clasificados como "Staphylococcus coagulasa positiva" (SCP). El grupo de SCP incluye a $S$. aureus, el SIG, S. lutrae y S. schleiferi subsp. coagulans, mientras que la producción de coagulasa de $S$. hyicus es variable $^{8,9}$

Si bien $S$. aureus es un frecuente colonizador en humanos y se encuentra ampliamente distribuido en hospederos mamíferos en general, las cepas del SIG se asocian predominantemente con perros y gatos ${ }^{10}$. Si bien en gatos Staphylococcus no son causantes frecuentes de enfermedades dermatológicas, estas bacterias pueden actuar como colonizantes de lesiones cutáneas y prolongando cuadros de dermatitis, foliculitis y piodermas superficiales. Además, es posible también aislarlo como el agente etiológico de estos cuadros ${ }^{11}$, los que requieren tratamiento antimicrobiano.

Así, en la última década algunos autores han sugerido que los gatos pueden ser reservorios de bacterias resistentes a antimicrobianos, basados en estudios de resistencia antimicrobiana en aislados clínicos de SCP obtenidos desde gatos y sus dueños en contacto estrecho ${ }^{12,13}$. Sin embargo, no existe información consistente de la distribución, diversidad y potencial multirresistencia de SCP en mascotas, ni el rol de ellas como reservorios de resistencia bacteriana. 
La resistencia a meticilina es una de las resistencias más relevantes a antimicrobianos que ha emergido en diferentes especies bacterianas. Meticilina fue el primer $\beta$-lactámico resistente a la acción de penicilinasas, introducida en la clínica práctica en $1959^{14}$. A pesar de poseer un espectro de acción más amplio y menos potencia que sus predecesores, su uso fue restringido casi exclusivamente al tratamiento de infecciones causadas por cepas de $S$. aureus productores de $\beta$-lactamasas. Desafortunadamente, las primeras cepas de $S$. aureus resistentes a meticilina (SARM) fueron identificadas casi inmediatamente después de su introducción en la práctica clínica ${ }^{15}$. Las cepas de SARM han sido consideradas tradicionalmente como patógenos intrahospitaliarios humanos (SARM-HA). Los factores de riesgo para la infección con SARM-HA incluyen hospitalización o cirugía reciente, permanencia por largo plazo en centros de atención médica, diálisis y el recibir de forma percutánea catéteres y dispositivos médicos ${ }^{16}$. Las cepas de SARM de origen nosocomial pueden transmitirse a la comunidad a través de los pacientes y sus contactos o del personal de salud; a pesar que también existe la probabilidad que estas cepas surjan "de novo", gracias a la adquisición de determinantes de resistencia por el genoma de una cepa de $S$. aureus previamente susceptible a meticilina ${ }^{17,18}$. Sin embargo; más recientemente, se han identificado casos de infección con cepas de SARM en personas sanas sin dichos factores de riesgo. Debido a que esas cepas son presumiblemente adquiridas en la comunidad, ellas son denominadas como SARM-adquirido en la comunidad (SARM-AC) ${ }^{16}$. Adicionalmente, desde el 2003 un tipo de SARM con una secuencia genómica específica emergió en animales de abasto y sus cuidadores, siendo denominada como SARM-asociado a ganado (MRSA-LA, por sus siglas en inglés) ${ }^{19,20}$. En Chile, Ledermann y cols ${ }^{21}$ informaron por primera vez cepas de SARM en 1967. Desde entonces, diversos autores han determinado una alta incidencia de infecciones por SARM, especialmente nosocomiales, lo que ha implicado un aumento en la morbilidad y mortalidad hospitalaria ${ }^{22,23}$.

El mecanismo que confiere resistencia a meticilina está codificado, principalmente, por el gen mecA, el cual produce una modificación en la proteína de unión a penicilina (PBP, por sus siglas en inglés) en la pared celular bacteriana, previniendo así la acción del fármaco. Por ello, las cepas de SARM deberían ser consideradas resistentes a todas las penicilinas, cefalosporinas, cefemes y otros $\beta$-lactámicos, como las combinaciones de ampicilina/sulbactam, amoxicilina/ácido clavulánico, ticarcilina/ácido clavulánico, piperacilina/tazobactam y tecarbapenemes, independiente de los resultados obtenidos in vitro con estos fármacos. Además, estos microorganismos pueden ser resistentes a los antimicrobianos más comúnmente usados, incluyendo aminoglucósidos, macrólidos, cloranfenicol, tetraciclinas y fluoroquinolonas ${ }^{24}$.
En personas hospitalizadas, SARM ha sido un problema desde la década de $1960^{25}$, siendo casi $20 \%$ de las septicemias en hospitales asociadas a $S$. aureus $^{26}$, de las cuales $64,4 \%$ fueron confirmadas como SARM en centros de cuidados intensivos en Estados Unidos de América en el año $2003^{27}$. Sin embargo, producto de diferentes programas de control y prevención, la incidencia de este patógeno a nivel intrahospitalario ha disminuido en $13 \%$ entre los años 2013 y $2014^{28}$.

Por otra parte, la información relativa a la resistencia a meticilina en cepas del SIG es escasa. No obstante, en los últimos años se ha identificado un número creciente de cepas de $S$. intermedius, $S$. pseudintermedius y $S$. schleiferi subsp. coagulans resistentes a meticilina ${ }^{29-32}$. Esto sugiere que los animales de compañía, como gatos y perros, pueden poseer cepas de SCP resistentes a meticilina, lo que puede suponer un problema preocupante para la medicina veterinaria, medicina humana y salud pública. Así, y para esclarecer esta situación, el desarrollo de estudios de resistencia bacteriana y su caracterización molecular podrán proveer la información necesaria para la implementación de medidas de control para enfrentar esta situación.

Por ello, este estudio incluyó como primer objetivo la determinación de los perfiles de susceptibilidad a antimicrobianos en cepas de SCP aisladas desde gatos sanos o con dermatopatías a los antimicrobianos más utilizados en la práctica clínica médico veterinaria, mientras que como segundo objetivo se incluyó el evaluar la presencia del gen mecA en las cepas de SCP, como base genética de la resistencia a meticilina.

\section{Materiales y Métodos}

\section{Protocolos de obtención de muestras}

Las muestras fueron obtenidas desde dos grupos de animales: gatos sanos $(n=66)$ y gatos con lesiones cutáneas $(n=68)$. Los individuos fueron recibidos en el Hospital Veterinario de la Facultad de Ciencias Veterinarias y Pecuarias (FAVET) de la Universidad de Chile, durante el año 2011. Los individuos del grupo de animales sanos concurrieron al hospital para consulta rutinaria o procedimientos médicos no relacionados con lesiones en piel (castraciones/esterilizaciones). Animales con lesiones cutáneas por sobre cuatro semanas, como placas eosinofílicas, úlceras, granulomas, dermatitis miliar y alopecia auto-inducida que no hubiesen recibido terapia antimicrobiana tópica y/o sistémica por al menos una semana antes a la toma de muestras, fueron incluidos en el segundo grupo. Los gatos sanos fueron muestreados en cinco zonas corporales: piel y pelaje de la zona frontal, mucosa bucal y gingival, mucosa anal, piel y pelaje de la zona inguinal, y piel y pelaje de la zona perianal; mientras que las muestras de los gatos con lesiones cutáneas 
fueron obtenidas desde sus lesiones. Cada zona o lesión fue frotada vigorosamente por $15 \mathrm{~s}$, con una tórula estéril previamente humedecida con solución salina ( $\mathrm{NaCl} 9 \%$ ) estéril, y luego depositada en medio de transporte Stuart $\left(\right.$ Oxoid $\left.^{\circledR}\right)$ para los análisis microbiológicos.

\section{Cepas bacterianas}

Todos los análisis de laboratorio fueron realizados en el Laboratorio de Bacteriología Clínica Veterinaria-FAVET, Universidad de Chile. A todas las muestras se les realizó tinción Gram para confirmar la presencia de cocáceas Gram positivas. Luego, las tórulas fueron incubadas en caldo tripticasa-soya $\left(\right.$ Difco $\left.^{\circledR}\right)$ suplementado con cloruro de sodio $10 \% \mathrm{p} / \mathrm{v}$, e incubadas por $24 \mathrm{~h}$ a $37^{\circ} \mathrm{C}$. Subsecuentemente, los caldos fueron sembrados en placas de agar sangre (5\% de sangre ovina) y sal-manitol $\left(\mathrm{BD}^{\circledR}\right) \mathrm{e}$ incubadas a $37^{\circ} \mathrm{C}$ por $24-48 \mathrm{~h}$. Las colonias obtenidas fueron analizadas en su morfología, presencia de hemólisis y color. Se seleccionaron colonias (un aislado por animal) circulares, blancas o grises, con hemólisis $\beta$ o caliente-fría y que crecieron en agar sal-manitol $\left(\mathrm{BD}^{\circledR}\right)$, lo que concuerda con las colonias de Staphylococcus spp. La presencia de Staphylococcus spp. en las muestras seleccionadas fue confirmada mediante la prueba de producción de catalasa $\left(\mathrm{Oxoid}^{\circledR}\right)$, y luego las cepas catalasa positivas fueron analizadas en su producción de coagulasa mediante la prueba de coagulasa en tubo. Finalmente, las cepas seleccionadas fueron identificadas a nivel de especie mediante el kit $\mathrm{BBL}^{\circledR} \mathrm{Crystal}^{\circledR}$ Gram-Positive ID $\left(\mathrm{BD}^{\circledR}\right)$, considerando un nivel de seguridad mayor al $95 \%$.

\section{Prueba de susceptibilidad a antimicrobianos}

Una vez ya identificadas las cepas, se determinó su susceptibilidad a antimicrobianos mediante el método de difusión en placa de Kirby-Bauer, de acuerdo al National Committee for Clinical Laboratory Standards ${ }^{33}$. Los antimicrobianos analizados incluyeron: oxacilina (en reemplazo de meticilina, $1 \mu \mathrm{g})$, ampicilina $(10 \mu \mathrm{g})$, amoxicilina/ác. clavulánico, $(30 \mu \mathrm{g})$ cefadroxilo $(30 \mu \mathrm{g})$, tetraciclina $(30 \mu \mathrm{g})$, doxiciclina $(30 \mu \mathrm{g})$, eritromicina $(15 \mu \mathrm{g})$, clindamicina $(2 \mu \mathrm{g})$, cotrimoxazol $(25 \mu \mathrm{g})$, enrofloxacina $(5 \mu \mathrm{g})$, ciprofloxacina $(5 \mu \mathrm{g})$ y vancomicina $(30 \mu \mathrm{g})$, utilizando discos comerciales $\left(\right.$ Oxoid $\left.^{\circledR}\right)$. La cepa de $S$. aureus ATCC 25923 fue utilizada como control de calidad de la prueba. Los resultados fueron expresados como porcentaje de sensibilidad y resistencia, considerando las cepas con sensibilidad intermedia como resistentes. Se consideró multirresistencia si se observó resistencia a tres o más antimicrobianos ${ }^{34}$.

\section{Extracción de ADN bacteriano y reacción de polimerasa en cadena (RPC)}

Para la detección del gen de resistencia a meticilina mecA, todas las cepas fueron sometidas a RPC. Primero, para la extracción del ADN bacteriano, se utilizó un kit comercial de extracción y purificación (Genomic DNA Purification Kit, Fermentas ${ }^{\circledR}$ ), siguiendo las instrucciones del fabricante. Luego, se utilizó el kit comercial PCR Master Mix 2X (Fermentas ${ }^{\circledR}$ ) para amplificar el ADN purificado. La mezcla de reacción de RPC incluyó 15 $\mu \mathrm{L}$ de Master Mix, $5 \mu \mathrm{L}$ de ADN bacteriano y $5 \mu \mathrm{L}$ de cada partidor. Los partidores utilizados fueron F: 5'-AAAATCGATGGTAAAGGTTGGC-3', y R: 5'-AGTTCTGCAGTACCGGATTTGC-3', con un tamaño molecular esperado del producto de $533 \mathrm{pb}^{35}$.

La amplificación del ADN se realizó en un termociclador Apollo (Thermocycler ${ }^{\mathbb{R}}$ ) siguiendo el protocolo descrito por Wichelhaus y cols ${ }^{35}$. Luego, y para visualizar los amplicones obtenidos, se realizó una electroforesis en gel de agarosa al $2 \%$ (Winkler ${ }^{\circledR}$ ) en tampón Tris-acetatoEDTA (TAE, Fermentas $\left.{ }^{\circledR}\right)$. Los productos de RPC $(5 \mu \mathrm{L})$ fueron mezclados con $1 \mu \mathrm{L}$ de $6 \mathrm{X}$ Mass Ruler Loading Dye Solution (Fermentas ${ }^{\circledR}$ ). La electroforesis se realizó a $90 \mathrm{~V}$ por $90 \mathrm{~min}$, utilizando el marcador de tamaño molecular Hyperladder ${ }^{\mathrm{TM}} 100 \mathrm{bp}\left(\right.$ Bioline $\left.^{\circledR}\right)$. Finalmente, el gel se incubó en bromuro de etidio $\left(0,5 \mathrm{mg} / \mathrm{mL}, \operatorname{Sigma}^{\circledR}\right)$ por $40 \mathrm{~min}$ y fotografiado en un transiluminador de luz UV (UVP transilluminator ${ }^{\mathbb{R}}$ ). Como control positivo se utilizó una cepa de SARM y como control negativo la cepa S. aureus ATCC 29213.

\section{Secuenciación y análisis de los productos amplificados}

Tres de los amplicones obtenidos fueron purificados utilizando el kit de extracción HiYield extraction PCR $\left(\right.$ Bioscience ${ }^{\circledR}$ ) y enviados a Genytec Ldta. para su secuenciación. Las secuencias fueron obtenidas utilizando el kit Big Dye Terminator (Life Technologies ${ }^{\circledR}$ ), mientras que la lectura de los fragmentos se realizó en el equipo ABI PRISM 310 Genetic Analyzer ${ }^{\circledR}$. Una vez obtenidas las secuencias, fueron alineadas en el programa ClustalW para obtener una secuencia de consenso. Finalmente, esta secuencia consenso fue alineada con la secuencia del gen mecA de la cepa SAMR252 disponible en GenBank ${ }^{\circledR}$ (número de acceso NC_002952.2) para obtener el porcentaje de identidad nucleotídica (PIN).

\section{Resultados}

Se recolectaron 134 muestras desde ambos grupos de animales (66 de gatos sanos y 68 de gatos con lesiones cutáneas). Desde estas muestras, se aislaron 72 cepas de SCP, 48 de gatos sanos y 24 de gatos con lesiones dermatológicas. De las cepas aisladas desde el primer grupo de animales, 11 correspondieron a $S$. aureus, 32 a $S$. intermedius y cinco a $S$. schleiferi subsp. coagulans; mientras que en los animales enfermos las cepas de 


\begin{tabular}{|c|c|c|c|c|c|c|c|c|c|c|}
\hline \multirow[t]{3}{*}{ Antimicrobiano } & \multicolumn{6}{|c|}{ Gatos sanos } & \multicolumn{4}{|c|}{ Gatos con dermatopatías } \\
\hline & \multicolumn{2}{|c|}{$\begin{array}{l}\text { S. intermedius } \\
(\mathrm{n}: 32)\end{array}$} & \multicolumn{2}{|c|}{$\begin{array}{l}\text { S. aureus } \\
(\mathrm{n}: 1)\end{array}$} & \multicolumn{2}{|c|}{$\begin{array}{l}\text { S. schleiferi subsp. coagulans } \\
\text { ( } \mathrm{n}: 5)\end{array}$} & \multicolumn{2}{|c|}{$\begin{array}{l}\text { S. intermedius } \\
\quad(n: 16)\end{array}$} & \multicolumn{2}{|c|}{$\begin{array}{l}\text { S. aureus } \\
\text { (n: } 8)\end{array}$} \\
\hline & $n$ & $\%$ & $\mathbf{n}$ & $\%$ & $\mathrm{n}$ & $\%$ & $n$ & $\%$ & $\mathrm{n}$ & $\%$ \\
\hline Amc & 3 & 9,4 & 0 & 0 & 2 & 40 & 3 & 18,8 & 0 & 0 \\
\hline Amp & 25 & 78,1 & 8 & 72,7 & 3 & 60 & 12 & 75 & 7 & 87,5 \\
\hline Oxa & 3 & 9,4 & 0 & 0 & 2 & 40 & 3 & 18,8 & 0 & 0 \\
\hline Cef & 3 & 9,4 & 0 & 0 & 2 & 40 & 4 & 25 & 0 & 0 \\
\hline Eri & 11 & 34,4 & 0 & 0 & 2 & 40 & 7 & 43,8 & 0 & 0 \\
\hline Cli & 3 & 9,4 & 0 & 0 & 2 & 40 & 5 & 31,3 & 1 & 12,5 \\
\hline Cip & 3 & 9,4 & 1 & 9,1 & 2 & 40 & 4 & 25 & 2 & 25 \\
\hline Enr & 4 & 12,5 & 0 & 0 & 2 & 40 & 4 & 25 & 1 & 12,5 \\
\hline Tet & 1 & 3,1 & 0 & 0 & 0 & 0 & 3 & 18,8 & 0 & 0 \\
\hline Dox & 2 & 6,3 & 0 & 0 & 0 & 0 & 3 & 18,8 & 0 & 0 \\
\hline SXT & 6 & 18,8 & 0 & 0 & 2 & 40 & 6 & 37,5 & 0 & 0 \\
\hline Van & 0 & 0 & 0 & 0 & 0 & 0 & 0 & 0 & 0 & 0 \\
\hline
\end{tabular}

Amc: amoxicilina-ácido clavulánico; Amp: ampicilina; Oxa: oxacilina; Cef: cefadroxilo; Eri: eritromicina; Cli: clindamicina; Cip: ciprofloxacina; Enr: enrofloxacina; Tet: tetraciclina; Dox: doxiciclina; SxT: cotrimoxazol; Van: vancomicina.

SCP correspondieron a ocho de $S$. aureus y 16 de $S$. intermedius.

La resistencia antimicrobiana detectada en estas cepas frente a cada uno de los antimicrobianos analizados se muestra en la Tabla 1, mientras que en la Tabla 2 se muestran los perfiles de resistencia de dicha cepas.

En relación a la detección del gen mecA, éste fue detectado en 11 de las 72 cepas procesadas (15,3\%). De estas 11 cepas, cinco (45,5\%) fueron aisladas desde gatos sanos y seis $(54,5 \%)$ desde gatos con lesiones cutáneas, y sólo ocho fueron resistentes fenotípicamente a oxacilina. En la Tabla 3 se encuentran los perfiles de resistencia antimicrobiana de las cepas donde se detectó este gen. Finalmente, la secuencia consenso obtenido de los amplicones secuenciados mostró 98\% de PIN al compararla con la secuencia del gen mecA de la cepa SAMR252 depositada en GenBank ${ }^{\circledR}$.

\section{Discusión}

Este estudio describe por primera vez en Chile la presencia de cepas de SCP poseedoras del gen mecA en mascotas (gatos), y también provee información detallada sobre la resistencia antimicrobiana de estas cepas a diferentes clases de antimicrobianos.

Del total de cepas aisladas, y con la metodología utilizada, $66,7 \%$ de las aisladas desde gatos sanos co- rrespondieron a $S$. intermedius, siendo la especie de SCP más frecuentemente aislada desde estos animales (32/48), concordando con lo reportado por otros autores ${ }^{30,31}$. De manera similar, en las muestras provenientes de gatos con dermatopatías, la especie de SCP predominante también correspondió a $S$. intermedius $(66,7 \%)$, lo que también concuerda con lo reportado internacionalmente ${ }^{4,36}$. No se aislaron otras especies de SCP desde las lesiones cutáneas, como $S$. schleiferi subsp. coagulans, el que también ha sido reportado en casos de pioderma en gatos ${ }^{37,38}$.

Un punto interesante de considerar respecto a la identificación de las especies de Staphylococcus, es la emergencia de nuevas especies como las pertenecientes al SIG. A la fecha, existen diversos reportes que indican que $S$. pseudintermedius y no $S$. intermedius es el principal patógeno responsable de enfermedades dermatológicas en gatos ${ }^{39}$. Debido a que el kit BBL Crystal ${ }^{\circledR}$ para Gram positivos utilizado en este estudio no es capaz de diferenciar entre dichas especies ${ }^{40}$, no fue posible diferenciar la presencia de $S$. pseudintermedius en los gatos muestreados, asumiéndose que los aislados identificados como $S$. intermedius corresponderían a $S$. pseudintermedius. Para ayudar a diferenciar ambas especies, pueden ser realizadas caracterizaciones moleculares como RFLP-RPC y RPC multiplex ${ }^{5,41}$. Por otra parte, el aislamiento de cepas de SCP desde gatos sanos ha sido reportado en diversos estudios $^{31,42}$, y también desde lesiones cutáneas de éstos ${ }^{31,43}$. Además, en los últimos años han aumentado los reportes 


\begin{tabular}{|c|c|c|c|c|c|c|}
\hline & n cepas & $\%$ & Perfil de resistencia & S. intermedius & S. aureus & S. schleiferi subsp. coagulans \\
\hline \multirow{10}{*}{$\begin{array}{l}\text { A } \\
(n: 48)\end{array}$} & 21 & 43,8 & Amp & 12 & 8 & 1 \\
\hline & 1 & 2,1 & Dox & 1 & 0 & 0 \\
\hline & 1 & 2,1 & Eri & 1 & 0 & 0 \\
\hline & 1 & 2,1 & Cip & 0 & 1 & 0 \\
\hline & 1 & 2,1 & Amp-SxT & 1 & 0 & 0 \\
\hline & 5 & 10,4 & Amp-Eri & 5 & 0 & 0 \\
\hline & 1 & 2,1 & Amp-Eri-SxT & 1 & 0 & 0 \\
\hline & 1 & 2,1 & Amp-Enr-Eri & 1 & 0 & 0 \\
\hline & 1 & 2,1 & Amp-Tet-Dox & 1 & 0 & 0 \\
\hline & 5 & 10,4 & Amp-Amc-Oxa-Cef-Eri-Enr-Cip-Cli-SxT & 3 & 0 & 2 \\
\hline \multirow{13}{*}{$\begin{array}{l}B \\
\text { (n: 24) }\end{array}$} & 7 & 29,2 & Amp & 4 & 3 & 0 \\
\hline & 1 & 4,2 & Eri & 1 & 0 & 0 \\
\hline & 1 & 4,2 & Cef & 1 & 0 & 0 \\
\hline & 1 & 4,2 & Amp-Eri & 1 & 0 & 0 \\
\hline & 1 & 4,2 & Amp-SxT & 1 & 0 & 0 \\
\hline & 2 & 8,3 & Amp-Cip & 0 & 2 & 0 \\
\hline & 1 & 4,2 & Amp-Cli & 0 & 1 & 0 \\
\hline & 1 & 4,2 & Amp-Enr & 0 & 1 & 0 \\
\hline & 1 & 4,2 & Amp-Eri-SxT & 1 & 0 & 0 \\
\hline & 1 & 4,2 & Amp-Tet-Dox-Cli & 1 & 0 & 0 \\
\hline & 1 & 4,2 & Amp-Enr-Cip-Eri-Cli-SxT & 1 & 0 & 0 \\
\hline & 1 & 4,2 & Amp-Amc-Oxa-Cef-Enr-Cip-Eri-Cli-SxT & 1 & 0 & 0 \\
\hline & 2 & 8,3 & Amp-Amc-Oxa-Cef-Enr-Cip-Tet-Dox-Eri-Cli-Sxt & 2 & 0 & 0 \\
\hline
\end{tabular}

Tabla 3. Perfiles de resistencia de las cepas de SCP aisladas desde gatos sanos (A) y desde gatos con dermatopatías (B) positivas a la detección del gen mecA

\begin{tabular}{|c|c|c|c|c|c|c|c|c|c|c|c|c|c|}
\hline & \multirow[t]{2}{*}{ Cepa } & \multicolumn{12}{|c|}{ Antimicrobianos } \\
\hline & & Ac & $\mathrm{Am}$ & Ox & $\mathrm{Ce}$ & Er & $\mathrm{Cl}$ & $\mathrm{Ci}$ & En & $\mathrm{Te}$ & Do & ST & Va \\
\hline \multirow[t]{5}{*}{ A } & S. schleiferi & $\mathrm{R}$ & $\mathrm{R}$ & $\mathrm{R}$ & $\mathrm{R}$ & $\mathrm{R}$ & $\mathrm{R}$ & $\mathrm{R}$ & $\mathrm{R}$ & $\mathrm{S}$ & S & $\mathrm{R}$ & S \\
\hline & S. intermedius & $\mathrm{R}$ & $R$ & $R$ & $\mathrm{R}$ & $\mathrm{R}$ & $\mathrm{R}$ & $\mathrm{R}$ & $\mathrm{R}$ & S & S & $\mathrm{R}$ & S \\
\hline & S. schleiferi & $\mathrm{R}$ & $R$ & $R$ & $\mathrm{R}$ & $\mathrm{R}$ & $\mathrm{R}$ & $\mathrm{R}$ & $\mathrm{R}$ & $\mathrm{S}$ & S & $R$ & S \\
\hline & S. intermedius & $\mathrm{R}$ & $\mathrm{R}$ & $\mathrm{R}$ & $\mathrm{R}$ & $\mathrm{R}$ & $\mathrm{R}$ & $\mathrm{R}$ & $\mathrm{R}$ & $S$ & S & $\mathrm{R}$ & S \\
\hline & S. intermedius & $\mathrm{R}$ & $\mathrm{R}$ & $\mathrm{R}$ & $\mathrm{R}$ & $\mathrm{R}$ & $\mathrm{R}$ & $\mathrm{R}$ & $\mathrm{R}$ & S & S & $\mathrm{R}$ & S \\
\hline \multirow[t]{6}{*}{ B } & S. intermedius & $\mathrm{R}$ & $R$ & $R$ & $\mathrm{R}$ & $\mathrm{R}$ & $\mathrm{R}$ & $R$ & $\mathrm{R}$ & $\mathrm{R}$ & $R$ & $\mathrm{R}$ & S \\
\hline & S. intermedius & $S$ & $R$ & S & $S$ & $\mathrm{R}$ & $S$ & $\mathrm{~S}$ & $S$ & $S$ & S & $\mathrm{S}$ & S \\
\hline & S. intermedius & $S$ & $\mathrm{R}$ & S & $S$ & $\mathrm{~S}$ & $S$ & $S$ & $S$ & $S$ & S & $S$ & S \\
\hline & S. intermedius & $R$ & $R$ & $R$ & $R$ & $R$ & $\mathrm{R}$ & $\mathrm{R}$ & $\mathrm{R}$ & S & S & $\mathrm{R}$ & S \\
\hline & S. intermedius & $\mathrm{R}$ & $R$ & $R$ & $S$ & $\mathrm{R}$ & $\mathrm{R}$ & $R$ & $\mathrm{R}$ & $S$ & S & $\mathrm{R}$ & S \\
\hline & S. aureus & $S$ & $\mathrm{R}$ & S & $S$ & $S$ & $S$ & $S$ & $S$ & S & $S$ & $S$ & S \\
\hline
\end{tabular}

Ac: amoxicilina/ácido clavulánico; Am: ampicilina; Ox: oxacilina; Ce: cefradoxilo; Er: eritromicina; Cl: clindamicina; Ci: ciprofloxacina; En: enrofloxacina; Te: tetraciclina; Do: doxiciclina; ST: cotrimoxazol; Va: vancomicina; S: sensible; R: resistente; SI: sensibilidad intermedia. 
de casos de infección con $S$. aureus, $S$. pseudintermedius y $S$. intermedius en personas y mascotas. Sumado a esto, nuevas especies de SCP han sido identificadas como zoonóticas ${ }^{10,31,38}$. Así, en años recientes ha emergido la infección por S. schleiferi subsp. coagulans, especialmente como una enfermedad ocupacional en médicos veterina$\operatorname{rios}^{38}$. En Chile, esta especie ha sido aislada y reportada como causa de otitis externa y pioderma en perros ${ }^{44}$. A la fecha no existen publicaciones a nivel nacional de la portación de SCP en gatos.

Por otra parte, la presencia de cepas resistentes a antimicrobianos en mascotas puede potencialmente comprometer a la salud pública, particularmente si estas cepas resistentes están presentes en individuos sanos que están en contacto con sus propietarios. La resistencia antimicrobiana más detectada en las cepas aisladas desde ambos grupos de animales fue frente a ampicilina. Estos resultados fueron esperables, debido a que diversos estudios han demostrado que cepas de SCP aisladas desde gatos se han vuelto cada vez más resistentes a penicilina y penicilinas semi-sintéticas como ampicilina ${ }^{45,46}$. Debido a que las penicilinas semi-sintéticas poseen múltiples usos terapéuticos en gatos, como en infecciones del tracto respiratorio anterior y enfermedades cutáneas ${ }^{47}$, esto podría estar promoviendo una alta presión de selección para dichos antimicrobianos ${ }^{48}$. Por el contrario, en este estudio no se detectaron cepas resistentes a vancomicina; más aún, considerando que este glicopéptido es sólo de uso intrahospitalario en medicina humana ${ }^{49}$, era esperable que ninguna cepa de origen animal expresase resistencia a este fármaco. En este contexto, han aumentado los reportes de $S$. aureus resistentes a vancomicina en el mundo ${ }^{50,51}$ y se está transformando en una preocupación emergente en medicina veterinaria ${ }^{52}$.

Con respecto a la resistencia a meticilina, en este estudio se detectaron ocho $(11,1 \%)$ cepas de SCP con esta resistencia fenotípica, correspondiendo seis de ellas a $S$. intermedius y dos a $S$. schleiferi subsp. coagulans. El primer reporte de cepas de SCP resistentes a meticilina en animales ocurrió en 1972, con una cepa de SARM aislada desde vacas con mastitis ${ }^{53}$, describiéndose posteriormente en numerosos reportes ${ }^{54,55}$. En medicina humana, la prevalencia de SARM ha sido monitoreada por décadas; sin embargo, la prevalencia en mascotas es difícil de estimar por varias razones: existencia de portadores asintomáticos, significativas diferencias geográficas y también porque las pruebas para la detección de SARM utilizada como control en este estudio, poseen baja sensibilidad para detectar $S$. intermedius resistentes a meticilina ${ }^{56,57}$. En este estudio no se aislaron cepas de SARM, a pesar de los niveles de detección reportados en la literatura científica, que alcanzan a $9 \%$.

La resistencia a meticilina se encuentra principalmente codificada por el gen mecA, el que se detectó en $8 / 8$
$(100 \%)$ de las cepas que demostraron esta resistencia fenotípica, y también en tres cepas sensibles a meticilina aisladas desde gatos con dermatopatías (12,5\%). En la mayoría de los casos, estas cepas junto con ser resistentes fenotípicamente a meticilina, también fueron resistentes al menos a ocho de los antimicrobianos analizados en el presente estudio. Esta multi-resistencia se encuentra ampliamente descrita en la literatura científica y puede deberse a la posición del gen mecA en el cassette cromosomal estafilocóccico mec ( $\mathrm{SCC} m e c$ ), el que generalmente porta genes de resistencia a varios otros antimicrobianos, como eritromicina, clindamicina y enrofloxacina ${ }^{46,59}$. El gen mecA forma parte del complejo genético mec en los elementos SCCmec. De este complejo se han descrito cuatro clases (A-D), los que se diferencian en su organización genética ${ }^{60}$. Estos elementos están formados por el gen $m e c \mathrm{~A}$, elementos reguladores y la recombinasa cromosomal del cassette $(c c r)$, la que se relaciona la integración/escisión sitio-específica de este elemento ${ }^{60,61}$. En este estudio, 3/11 cepas positivas al gen mecA fueron susceptibles a meticilina, lo que se puede explicar por la existencia de cuatro clases de expresión fenotípica de resistencia a este antimicrobiano en $S$. aureus $^{62,63}$.

Estos hallazgos, sumado a otros reportes, indican un incremento en la frecuencia de detección del gen mecA en cepas de SCP aisladas desde gatos. Además, el incremento en la prevalencia de cepas del SIG resistentes a meticilina y su potencial zoonótico constituyen un elemento de creciente interés para la práctica clínica de animales menores en medicina veterinaria y para la salud pública ${ }^{30,64}$, situación que debiese enfrentarse según las indicaciones de FAO-OMS-OIE bajo el concepto de "Una Salud" 65 .

\section{Conclusiones}

Los resultados obtenidos en el presente estudio constituyen la primera evidencia nacional del rol de las mascotas como reservorio de cepas de Staphylococcus coagulasa positiva que podrían representar un riesgo para la salud pública, al exhibir altos patrones de resistencia y albergar genes codificantes de resistencia a fármacos tan relevantes como el gen mecA.

Agradecimientos. Los autores agradecen a María Teresa Ulloa (Programa de Microbiología y Micología, Instituto de Ciencias Biomédicas, Facultad de Medicina, Universidad de Chile) por su gentil donación de la cepa de SARM utilizada en este estudio.

\section{Resumen}

Introducción: La resistencia bacteriana constituye un tema de preocupación para la salud pública mundial. Últimamente han aumentado los reportes de resistencia 
a antimicrobianos, incluida meticilina, en cepas de Staphylococcus coagulasa positiva (SCP) aisladas desde mascotas. Sin embargo, en Chile esta información es escasa. Objetivos: Determinar el perfil de susceptibilidad antimicrobiana y detectar el gen mecA en cepas de SCP aisladas desde gatos en Chile. Materiales y Métodos: Se obtuvieron 134 muestras desde gatos sanos y con lesiones dermatológicas. Las cepas fueron caracterizadas en $\mathrm{su}$ producción de coagulasa e identificadas mediante kit BBL Crystal. La susceptibilidad antimicrobiana se determinó mediante el método de Kirby Bauer ante 12 antimicrobia- nos, incluida oxacilina. Todas las cepas fueron sometidas a RPC para la detección del gen mecA. Resultados: 72 cepas de SCP fueron aisladas, incluyendo $S$. aureus y $S$. intermedius. Se detectó resistencia antimicrobiana a al menos un antimicrobiano en cepas de gatos sanos (75\%) y de gatos con lesiones cutáneas $(87,5 \%)$. El gen mecA fue detectado en ocho cepas resistentes a meticilina y en tres cepas sensibles, siendo en general multi-resistentes. Discusión: Estos resultados destacan el rol de las mascotas como reservorios de resistencia bacteriana y su potencial impacto en la salud pública.

\section{Referencias bibliográficas}

1.- Guardabassi L, Prescott J F. Antimicrobial stewardship in small animal veterinary practice: from theory to practice. Vet Clin North Am Small Anim Pract 2015; 45: 361-76.

2.- Malik S, Peng H, Barton M. Antibiotic resistance in staphylococci associated with cats and dogs. J Appl Microbiol 2005; 99: 1283-93.

3.- Cohn L, Middleton J. A veterinary perspective on methicillin-resistant staphylococci. J Vet Emerg Crit Care 2010; 20: 31-45.

4.- Bond R, Loeffler A. What's happened to Staphylococcus intermedius? Taxonomic revision and emergence of multi-drug resistance. J Small Anim Pract 2012; 53: 147-54.

5.- Blaiotta G, Fusco V, Ercolini D, Pepe O, Coppola S. Diversity of Staphylococcus species strains based on partial kat (catalase) gene sequences and design of a PCR-restriction fragment length polymorphism assay for identification and differentiation of coagulasepositive species ( $S$. aureus, $S$. delphini, $S$ hyicus, $S$. intermedius, $S$. pseudintermedius, and S. schleiferi subsp. coagulans). J Clin Microbiol 2010; 48: 192-201.

6.- Devriese L, Vancanneyt M, Baele M, Vaneechoutte M, De Graef E, Snauwaert C, et al. Staphylococcus pseudintermedius sp. nov., a coagulase-positive species from animals. Int J Syst Evol Microbiol 2005; 55: 1569-73.

7.- Bannoehr J, Guardabassi L. Staphylococcus pseudintermedius in the dog: taxonomy, diagnostics, ecology, epidemiology and pathogenicity. Vet Dermatol 2012; 23: 253-66.

8.- Kloos W, Schleifer K. Genus IV: Staphylococcus. Sneath, PHA, Mair NS, Sharpe ME, Holt JG, editors. Bergey's Manual of Systematic Bacteriology, 1st ed. Baltimore, Williams and Wilkins; 1986, p. 1013-1035.

9.- Foster G, Roos H, Hutson R, Collins M. Staphylococcus lutrae sp. nov, a new coagulase positive species isolated from otters. Int J Syst Bacteriol 1997; 47: 724-26.

10.- Walther B, Hermes J, Cuny C, Wieler L H, Vincze S, Elnaga YA, et al. Sharing more than friendship-nasal colonization with coagulasepositive staphylococci (CPS) and co-habitation aspects of dogs and their owners. PLoS One 2012; 7: 1-7.

11.- Malik S, Christensen H, Peng H, Barton M. Presence and diversity of the $\beta$-lactamase gene in cat and dog staphylococci. Vet Microbiol 2007; 123: 162-8.

12.- Weese J, van Duijkeren E. Methicillin-resistant Staphylococcus aureus and Staphylococcus pseudintermedius in veterinary medicine. Vet Microbiol 2006; 140: 418-29.

13.- Gandolfi-Decristophoris P, Regula G, Petrini O, Zinsstag J, Schelling E. Prevalence and risk factors for carriage of multi-drug resistant Staphylococci in healthy cats and dogs. J Vet Sci 2013; 14: 449-56.

14.- Moellering R C, Ferraro M J. Introduction: solving the clinical problem of vancomycin resistance. Clin Infectious Dis 2012; 54: 201-2.

15.- De Leo F, Otto M, Kreiswirth B, Chambers H. Community-associated methicillin-resistant Staphylococcus aureus. Lancet 2010; 375: 1557-68.

16.- Vandenesch F, Naimi T, Enright M C, Lina G, Nimmo G R, Heffernan H, et al. Communityacquired methicillin-resistant Staphylococcus aureus carrying Panton-Valentine leukocidin genes: worldwide emergence. Emerg Infect Dis 2003; 9: 978-84

17.- Nour M, Mastouri M, Ben Nejma M. Methicillin resistance in Staphylococcus aureus; emergence and molecular basis. Pathol Biol (París) 2005; 53: 334-40.

18.- Durand G, Bes M, Meugnier H, Enright M C, Forey F, Liassine N, et al. Detection of new methicillin-resistant Staphyloccus aureus clones containing the toxic shock syndrome toxin 1 gene, responsible for hospital-and communityacquired infections in France. J Clin Microbiol 2006; 44: 847-53.

19.- Van Loo I, Huijsdens X, Tiemersma E, de Neeling A, van de Sande-Bruinsma N, Beaujean D, et al. Emergence of methicillinresistant Staphylococcus aureus of animal origin in humans. Emerg Infect Dis 2007; 13: 1834.
20.- Smith T, Male M, Harper A, Kroeger J S, Tinkler G P, Moritz E D, et al. Methicillinresistant Staphylococcus aureus (MRSA) strain ST398 is present in midwestern US swine and swine workers. PloS One 2009; 4: 1-6.

21.- Ledermann W, Mímica I. Sensibilidad in vitro del $S$. aureus a meticilina y cloxacilina en los últimos ocho años (1961-1968). Rev Chil Pediatr 1970; 41: 213.

22.- Ulloa M, Porte L, Carmi K, Varela C, Fica A. Comparación de reacción de polimerasa en cadena, látex y antibiograma para detección de Staphylococcus aureus meticilina resistente. Rev Chilena Infectol 2001; 18: 255-60.

23.- Gil M. Staphylococcus aureus: Microbiología y aspectos moleculares de la resistencia a meticilina. Rev Chilena Infectol 2000; 17: 145-52.

24.- Türkyilmaz S, Tekbiyik S, Oryasin E, Bozdogan B. Molecular epidemiology and antimicrobial resistance mechanisms of methicillin-resistant Staphylococcus aureus isolated from bovine milk. Zoonoses Public Health 2010; 57: 197-203.

25.- Boyce J M. Methicillin-resistant Staphylococcus aureus in hospitals and long-term care facilities: microbiology, epidemiology, and preventive measures. Infect Control Hosp Epidemiol 1992;13: 725-37.

26.- Wisplinghoff H, Bischoff T, Tallent S M, Seifert H, Wenzel R P, Edmond M B. Nosocomial bloodstream infections in US hospitals: analysis of 24,179 cases from a prospective nationwide surveillance study. Clin Infect Dis 2004; 39: 309-17.

27.- Klevens R M, Edwards J R, Tenover F C, McDonald L C, Horan T, Gaynes R. Changes in the epidemiology of methicillin-resistant Staphylococcus aureus in intensive care units in U.S. hospitals, 1992-2003. Clin Infect Dis 2006; 42: 389-91.

28.- CDC, Centers for Disease Control and Prevention. Healthcare associated infections progress report. 2016. http:/www.cdc.gov/hai/ pdfs/stateplans/factsheets/us.pdf. [Consultado el 14 de mayo de 2016]. 
29.- Kawakami T, Shibata S, Murayama N, Nagata M, Nishifuji K, Iwasaki T, et al. Antimicrobial susceptibility and methicillin resistance in Staphylococcus pseudintermedius and Staphylococcus schleiferi subsp. coagulans isolated from dogs with pyoderma in Japan. $\mathrm{J}$ Vet Med Sci 2010; 72: 1615-9.

30.- Perreten V, Kadlec K, Schwarz S, Grönlund-Andersson U, Finn M, Greko C, et al. Clonal spread of methicillin-resistant Staphylococcus pseudintermedius in Europe and North America: an international multicentre study. J Antimicrob Chemother 2010; 65: 1145-54.

31.- van Duijkeren E, Catry B, Greko C, Moreno M A, Pomba M C, Pyörälä S, et al. Review on methicillin-resistant Staphylococcus pseudintermedius. J Antimicrob Chemother 2011; 66: 2705-14.

32.- Couto N, Belas A, Couto I, Perreten V, Pomba C. Genetic relatedness, antimicrobial and biocide susceptibility comparative analysis of methicillin-resistant and-susceptible Staphylococcus pseudintermedius from Portugal. Microb Drug Resist 2014; 20: 364-71.

33.- National Committe for Clinical Laboratory Standards (NCCLS). Performance standards for antimicrobial disk susceptibility testing. Twenty-first informational supplement. NCCLS document M100-S21. National Committe for Clinical Laboratory Standards 2011.

34.- Magiorakos A P, Srinivasan A, Carey R B, Carmeli Y, Falagas M E, Giske C G, et al. Multidrug-resistant, extensively drug-resistant and pandrug-resistant bacteria: an international expert proposal for interim standard definitions for acquired resistance. Clin Microbiol Infect 2012; 18: 268-81.

35.- Wichelhaus T, Kern S, Schafer V, Brade V. Rapid detection of epidemic strains of methicillin-resistant Staphylococcus aureus. J Clin Microbiol 1999; 37: 690-3.

36.- Kottler S, Middleton J, Perry J, Weese J S, Cohn L A. Prevalence of Staphylococcus aureus and methicillin-resistant Staphylococcus aureus carriage in three populations. J Vet Intern Med 2010; 24: 132-9.

37.- Griffeth G, Morris D, Abraham J, Shofer F S, Rankin S C. Screening for skin carriage of methicillin-resistant coagulase-positive staphylococci and Staphylococcus schleiferi in dogs with healthy and inflamed skin. Vet Dermatol 2008; 19: 142-9.

38.- Morris D, Boston R, O'Shea K, Rankin S. The prevalence of carriage of methicillinresistant staphylococci by veterinary dermatology practice staff and their respective pets. Vet Dermatol 2010; 21: 400-7.

39.- Kadlec K, Schwarz S, Perreten V, GrönlundAndersson U, Finn M, Greko C, et al. Molecular analysis of methicillin-resistant Staphylococcus pseudintermedius of feline origin from different
European countries and North America. J Antimicrob Chemother 2010; 65: 1826-8.

40.- Sasaki T, Kikuchi K, Tanaka Y, Takahashi N, Kamata S, Hiramatsu K. Methicillin-resistant Staphylococcus pseudintermedius in a veterinary teaching hospital. J Clin Microbiol 2007; 45: 1118-25.

41.- Sasaki T, Tsubakishita S, Tanaka Y, Sakusabe A, Ohtsuka M, Hirotaki S, et al. Multiplex-PCR method for species identification of coagulasepositive staphylococci. J Clin Microbiol 2010; 48: 765-9.

42.- Rubin J, Chirino-Trejo M. Prevalence, sites of colonization, and antimicrobial resistance among Staphylococcus pseudintermedius isolated from healthy dogs in Saskatoon, Canada. J Vet Diagn Invest 2011; 23: 351-4.

43.- Weese J S, Dick H, Willey B M, McGeer A, Kreiswirth B N, Innis B, et al. Suspected transmission of methicillin-resistant Staphylococcus aureus between domestic pets and humans in veterinary clinics and in the household. Vet Microbiol 2006; 115: 148-55.

44.- Muñoz L, Molina M, Heresmann M, Abusleme F, Ulloa M T, Borie C, et al. First report of isolation of Staphylococcus schleiferi subspecies coagulans from dogs with pyoderma and external otitis in Chile. Arch Med Vet 2012; 44: 261-5.

45.- Argudin M, Tenhagen B, Fetsch A, Sachsenröder J, Käsbohrer A, Schroeter J A, et al. Virulence and resistance determinants in German Staphylococcus aureus ST398 isolates from non-human origin. Appl Environ Microbiol 2011; 77: 3052-60.

46.- Nienhoff U, Kadlec K, Chaberny I, Verspohl J, Gerlach G F, Kreienbrock L, et al. Methicillinresistant Staphylococcus pseudintermedius among dogs admitted to a small animal hospital. Vet Microbiol 2011; 150: 191-7.

47.- Hill P B, Lo A, Eden C A N, Huntley S, Morey V, Ramsey S, et al. Survey of the prevalence, diagnosis and treatment of dermatological conditions in small animals in general practice. Vet Rec 2006; 158: 533-9.

48.- Six R, Cleaver D, Lindeman, C J, Cherni J, Chesebrough R, Papp G, et al. Effectiveness and safety of cefovecin sodium, an extendedspectrum injectable cephalosporin, in the treatment of cats with abscesses and infected wounds. J Am Vet Med Assoc 2009; 234: 81-7.

49.- Vandecasteele S, de Vriese A. Recent changes in vancomycin use in renal failure. Kidney Int 2010; 77: 760-4.

50.- Saravolatz L, Pawlak J, Johnson L. In vitro activity of ceftaroline against communityassociated methicillin-resistant, vancomycinintermediate, vancomycin-resistant, and daptomycin-non susceptible Staphylococcus aureus isolates. Antimicrob Agents Chemother 2010; 54: 3027-30
51.- Melo-Cristino J, Resina C, Manuel V, Lito L, Ramírez M. First case of infection with vancomycin-resistant Staphylococcus aureus in Europe. Lancet 2013; 382: 205.

52.- Ortega C, Simón M. Staphylococcus aureus resistentes a meticilina (MRSA) y a otros $\beta$-lactámicos en animales de compañía (perro y gato); aproximación a la situación actual y al riesgo para la salud pública. Vet Arg 2010; 27: $1-23$.

53.- Devriese L, van Damme L, Fameree L. Methicillin (cloxacillin)-resistant Staphylococcus aureus strains isolated from bovine mastitis cases. Zentralbl Veterinär Med B 1972; 19: 598-605.

54.- García-Álvarez L, Holden M, Lindsay H, Webb C R, Brown D F J, Curran M D, et al. Meticillin-resistant Staphylococcus aureus with a novel mecA homologue in human and bovine populations in the UK and Denmark: a descriptive study. Lancet Infect Dis 2011; 11 : 595-603.

55.- Vincze S, Stamm I, Kopp P, Hermes J, Adlhoch C, Semmler T, et al. Alarming proportions of methicillin-resistant Staphylococcus aureus (MRSA) in wound samples from companion animals, Germany 2010-2012. PloS One 2014; 9: 13-9.

56.- Guardabassi L, O’Donoghue M, Moodley A, Ho J, Boos M. Novel lineage of methicillinresistant Staphylococcus aureus, Hong Kong. Emerg Infect Dis 2009; 15: 1998-2000.

57.- Morris D, Lautenbach E, Zaoutis T, Leckerman K, Edelstein P H, Rankin S C. Potential for pet animals to harbour methicillinresistant Staphylococcus aureus when residing with human MRSA patients. Zoonoses Public Health 2012; 59: 286-93.

58.- Loeffler A, Lloyd D. Companion animals: a reservoir for methicillin-resistant Staphylococcus aureus in the community? Epidemiol Infect 2010; 138: 595-605.

59.- Castellano M, Perozo J, Parra A, Ginestre M M, Rincón G C. Antimicrobial susceptibility and polyclonal dissemination of Staphylococcus aureus strains. Rev Chilena Infectol 2014; 31: 165-72.

60.- Shore A, Deasy E, Slickers P, Brennan G, O'Connell B, Monecke S, et al. Detection of staphylococcal cassette chromosome mec type $\mathrm{XI}$ carrying highly divergent $m e c \mathrm{~A}, m e c \mathrm{I}$, $m e c \mathrm{R} 1$, blaZ, and $c c r$ genes in human clinical isolates of clonal complex 130 methicillinresistant Staphylococcus aureus. Antimicrob Agents Chemother 2011; 55: 3765-73.

61.- International Working Group on the Classification of Staphylococcal Cassette Chromosome Elements (IWG-SCC). Classification of staphylococcal cassette chromosome mec (SCCmec): guidelines for reporting novel SCCmec elements. Antimocrob Agents Chemother 2009; 53: 4961-7. 
62.- Kim C, Mwangi M, Chung M, Milheirço C, de Lencastre H, Tomasz A. The mechanism of heterogeneous beta-lactam resistance in MRSA: key role of the stringent stress response. PLoS One 2013; 8: 1-10.

63.- Dordel J, Kim C, Chung M, de la Gándara M P, Holden M T J, Parkhill J, et al. Novel determinants of antibiotic resistance: identification of mutated loci in highly methicillin-resistant subpopulations of methicillin-resistant Staphylococcus aureus. Mbio 2014; 5: 1-9.

64.- Graveland H, Duim B, van Duijkeren E, Heederik D, Wagenaar JA. Livestockassociated methicillin-resistant Staphylococcus aureus in animals and humans. Int J Med Microbiol 2011; 301 : 630-4.
65.- FAO-Food and Agriculture Organization of the United Nations, OIE-International Organization for Animal Health, WHO (OMS)World Health Organization. Antimicrobial resistance: a manual for developing national action plans. 2016. http://apps.who.int/iris/ bitstream/10665/204470/1/9789241549530 eng.pdf?ua $=1$. (Consultado el 15 de marzo de 2016) 